\title{
Atypical modulation of distant functional connectivity by cognitive state in children with Autism Spectrum Disorders
}

\author{
Xiaozhen You ${ }^{1 *}$, Megan Norr ${ }^{1}$, Eric Murphy ${ }^{1}$, Emily S. Kuschner ${ }^{2}$, Elgiz Bal' ${ }^{2}$, William D. Gaillard ${ }^{2}$, \\ Lauren Kenworthy ${ }^{2}$ and Chandan J. Vaidya ${ }^{1,2 *}$
}

Department of Psychology, Georgetown University, Washington, DC, USA

${ }^{2}$ Children's Research Institute, Children's National Medical Center, Washington DC, USA

\section{Edited by:}

Ralph-Axel Müller, San Diego State

University, USA

Reviewed by:

Christopher Monk, University

Hospitals Bristol NHS Foundation

Trust, UK

Brandon Keehn, Children's Hospital

Boston, USA

\section{${ }^{*}$ Correspondence:}

Xiaozhen You and Chandan J.

Vaidya, Department of Psychology,

Georgetown University, 306

White-Gravenor, Washington, DC

20057, USA

e-mail: xy34@georgetown.edu:

cjv2@georgetown.edu
We examined whether modulation of functional connectivity by cognitive state differed between pre-adolescent children with Autism Spectrum Disorders (ASD) and age and IQ-matched control children. Children underwent functional magnetic resonance imaging (fMRI) during two states, a resting state followed by a sustained attention task. A voxel-wise method was used to characterize functional connectivity at two levels, local (within a voxel's $14 \mathrm{~mm}$ neighborhood) and distant (outside of the voxel's $14 \mathrm{~mm}$ neighborhood to the rest of the brain) and regions exhibiting Group $\times$ State interaction were identified for both types of connectivity maps. Distant functional connectivity of regions in the left frontal lobe (dorsolateral [BA 11, 10]; supplementary motor area extending into dorsal anterior cingulate [BA 32/8]; and premotor [BA 6, 8, 9]), right parietal lobe (paracentral lobule [BA 6]; angular gyrus [BA 39/40]), and left posterior middle temporal cortex (BA 19/39) showed a Group $\times$ State interaction such that relative to the resting state, connectivity reduced (i.e., became focal) in control children but increased (i.e., became diffuse) in ASD children during the task state. Higher state-related increase in distant connectivity of left frontal and right angular gyrus predicted worse inattention in ASD children. Two graph theory measures (global efficiency and modularity) were also sensitive to Group $\times$ State differences, with the magnitude of state-related change predicting inattention in the ASD children. Our results indicate that as ASD children transition from an unconstrained to a sustained attentional state, functional connectivity of frontal and parietal regions with the rest of the brain becomes more widespread in a manner that may be maladaptive as it was associated with attention problems in everyday life.

Keywords: fMRI, intrinsic, spontaneous, task, ASD

\section{INTRODUCTION}

Disturbed functional connectivity across distant regions is posited to mediate functional impairment in Autism Spectrum Disorders (ASD). Functional impairment in ASD comprises symptoms of ASD (e.g., difficulty with social interaction and communication, repetitive and restricted behaviors and interests) as well as problems with executive function, the goal-directed regulation of attention, actions and thoughts (Hill, 2004; Kenworthy et al., 2005, 2008). While executive dysfunction is not part of ASD diagnosis, it is associated with symptom presentation (e.g., Lopez et al., 2005; Kenworthy et al., 2009; Yerys et al., 2009a) and decreased independence and poor outcomes in adulthood [see review Hume et al. (2009)]. An emerging theoretical view of ASD is that frontal-posterior temporal synchronization of bloodoxygen level dependent (BOLD) signal is reduced in ASD subjects while they are engaged in social/communicative or executive functions (Just et al., 2012). Such "underconnectivity" has also been observed in spontaneous low-frequency BOLD fluctuations while subjects are not engaged in a directed task, a state of unconstrained cognition that is referred to as "resting" (Cherkassky et al., 2006; Kennedy and Courchesne, 2008; Assaf et al., 2010;
Weng et al., 2010; Wiggins et al., 2011; Gotts et al., 2012; Von dem Hagen et al., 2012). In addition to evidence supporting underconnectivity in ASD, greater than normal functional connectivity ("overconnectivity") has also been noted, either across cortical regions or between subcortical and cortical regions, during taskevoked (Noonan et al., 2009; Shih et al., 2011; Lai et al., 2012) as well as resting (Monk et al., 2009; Di Martino et al., 2011) states. Cognitive conditions that yield abnormally weaker or stronger functional connectivity in ASD are currently not well understood (Müller et al., 2011).

Functional connectivity may be atypical in ASD not only with respect to overall strength but also in its modulation by cognitive state. Studies of healthy adults show that the topology of functional network organization is remarkably similar during task-evoked and resting states. Networks delineated from spontaneous BOLD fluctuations while subjects rest (termed intrinsic connectivity networks) conform to activation patterns observed during visual, auditory, sensorimotor, executive, and self/internally-oriented tasks (Smith et al., 2009) and predict individual differences in task-evoked activation and associated performance (Fox et al., 2006; Mennes et al., 2010; 
Gordon et al., 2012c). Further, intrinsic connectivity networks are preserved during sleep (Fukunaga et al., 2006) and light anesthesia (Vincent et al., 2007; Greicius et al., 2008), suggesting that they do not depend upon conscious cognition. While their topology is preserved across states, their strength differs in several ways: First, intrinsic connectivity was stronger, within networks and in anticorrelation across networks, during awake than non-conscious states [see review Heine et al. (2012)]. Second, within-subjects' comparison showed that functional connectivity became stronger from resting to a task-evoked state selectively, in regions activated during the task such as auditory (Arfanakis et al., 2000), visual (Arfanakis et al., 2000; Hampson et al., 2004; Nir et al., 2006), or motor (Arfanakis et al., 2000; Jiang et al., 2004). Third, functional connectivity decreased across some networks during task performance relative to a resting state (Fransson, 2006; Gordon et al., 2012b), suggesting that specific networks became more segregated when subjects were in a cognitive state constrained by a task. Fourth, the extent to which functional connectivity changed from resting to task states, particularly across networks, varied across individuals based upon dopamine neurotransmitter function and traits of distractibility and impulsivity (Gordon et al., 2012b). Together, these findings support the notion that functional connectivity is dynamic, and its modulation by cognitive state is associated with individual variability in attentional function. Whether state-related changes in functional connectivity are atypical in ASD and whether they predict attentional function is unknown.

The goal of the present study was to examine whether changes in functional connectivity, from a resting to a sustained attention state differ between ASD and typically developing (control) 9-13 year-old children. We focused on this narrow age range later in childhood in order to minimize developmental differences and maximize chances of acquiring two motion-free back-to-back fMRI runs from each child. We measured the strength of functional connectivity using a voxel-wise method that distinguished local connectivity, defined as within a voxel's $14 \mathrm{~mm}$ neighborhood, and distant connectivity, defined as connectivity of a voxel to the rest of the brain, outside of its $14 \mathrm{~mm}$ neighborhood. Such a voxel-wise data-driven method allows testing predictions without regard to a priori functional divisions, an approach that distinguishes the present study from past functional connectivity studies of ASD. For distant connectivity, we predicted a Group $\times$ State interaction such that control but not ASD children would modulate connectivity in response to the sustained attention state. As adult findings reviewed above showed that connectivity of selective networks became stronger during a task relative to a resting state, we reasoned that in control children, such a change suggestive of focal connectivity networks (i.e., task-relevant connections get stronger while task-irrelevant connections get weaker) ought to be expressed as a net reduction in our estimate of distant connectivity, which considers all voxels in the brain. In contrast, in light of the many underconnectivity findings in ASD during both task-evoked and resting states reviewed above, we expected overall weaker distant connectivity and little change from resting to task states. Further, we also explored whether whole-brain metrics of connectivity using two graph theory measures, global efficiency and modularity, would be sensitive to Group $\times$ State interaction. Global efficiency, measured by path length and reflecting network integration, characterizes the average "speed" of information transfer between any pair of nodes (Latora and Marchiori, 2001; Achard and Bullmore, 2007), and was lower in ASD subjects in a resting state magnetoencephalography study (Tsiaras et al., 2011). Modularity, on the other hand, reflects network segregation, through defining how well an entire network is organized into modules of densely interconnected nodes (Newman, 2006), and was higher in ASD subjects in a resting state electroencephalography study (Barttfeld et al., 2011). For regions (and graph theory metrics) showing the predicted interaction, we examined whether the state-related change in functional connectivity was related to attention problems measured by the inattention score of the ADHD Rating Scale (DuPaul et al., 1998). We focused upon attention, rather than hyperactivity/impulsivity or ASD symptoms, as it is most closely related to sustained attention, the task-state examined here. Due to the lack of past work on local connectivity changes by state in healthy or ASD adults or children, we tested for the same Group $\times$ State interaction but made no predictions.

\section{METHODS \\ SUBJECTS}

Thirty-one children aged 9-13 years, 15 with a diagnosis of ASD ( 3 left handed and 12 right handed) and 16 control children (all right handed), matched for age, IQ, and gender (see Table 1), participated in the study after complying with consenting guidelines of the Georgetown University and Children's National Medical

Table 1 | Demographic characteristics (Mean and standard deviation in parenthesis).

\begin{tabular}{|c|c|c|}
\hline & ASD & Control \\
\hline$N$ & 15 & 16 \\
\hline $\begin{array}{l}\text { Gender (females) }\left(\chi^{2}=1.06\right. \\
p=0.30)\end{array}$ & 3 & 7 \\
\hline Age (in years) $(p=0.96)$ & $11.2(1.4)$ & $11.2(1.3)$ \\
\hline Full scale IO ( $p=0.26)$ & $118.7(11.5)$ & $123.0(9.2)$ \\
\hline Performance IQ $(p=0.17)$ & $112.7(12.9)$ & $118.5(9.8)$ \\
\hline Verbal IQ $(p=0.44)$ & $120.4(11.3)$ & $123.5(11.0)$ \\
\hline $\begin{array}{l}\text { ADHD Rating scale inattentive } \\
\text { raw score }(0-25)(p<0.0001)\end{array}$ & $13.9(6.2)$ & $4.1(3.5)$ \\
\hline $\begin{array}{l}\text { ADHD Rating scale } \\
\text { hyperactive/impulsive raw score } \\
(0-25)(p<0.001)\end{array}$ & $8.6(5.5)$ & $2.4(3.0)$ \\
\hline ADOS Communication total (1-7) & $3.0(1.8)$ & - \\
\hline $\begin{array}{l}\text { ADOS Social interaction total } \\
(2-13)\end{array}$ & $7.5(3.1)$ & - \\
\hline $\begin{array}{l}\text { ADOS Stereotypical behaviors } \\
\text { and restricted interests total }(0-5)\end{array}$ & $1.8(1.7)$ & - \\
\hline ADI-R Total verbal score (7-24) & $16.0(4.8)$ & - \\
\hline $\begin{array}{l}\text { ADI-R Total social interaction } \\
\text { score (11-28) }\end{array}$ & $19.9(5.5)$ & - \\
\hline ADI-R Restrictive interests and & $4.9(1.3)$ & - \\
\hline
\end{tabular}

repetitive behaviors score (3-7) 
center Institutional Review Boards. This sample was retained after applying criteria for head motion, from a total sample of 24 ASD and 26 control children. ASD children were recruited through the Center for ASD at Children's National Medical Center. Control children were recruited from the Washington DC area community through advertisements at public venues and pediatrician offices.

ASD case classification followed diagnosis by a trained and experienced clinician based on the DSM-IV-TR criteria (American Psychiatric Association, 2000) and was confirmed with the Autism Diagnostic Interview-Revised (ADI-R) (Lord et al., 1994) and the Autism Diagnostic Observation ScheduleGeneric (ADOS-G) (Lord et al., 2000) following the criteria established by the NICHD/NIDCD Collaborative Programs for Excellence in Autism (Lainhart et al., 2006). These criteria require that the child meet ADI-R cutoff for autism in the social domain and at least one other domain (communication and/or repetitive behaviors and restricted interests), and meet ADOS cutoff (autism or ASD) for the combined social and communication score. One ASD subject met criteria for an ASD diagnosis on the $\mathrm{ADI}$ and ADOS, and by clinical diagnosis two years prior to this study, but on re-evaluation showed significant improvement on the ADOS.

Exclusion criteria included: (1) Full-Scale IQ below 80 as measured by the Wechsler Intelligence Scale for Children (WISC-IV) or Wechsler Abbreviated Scale of Intelligence (WASI) (Wechsler, 1999); (2) Other neurological diagnosis(e.g., epilepsy) based on parent report; (3) Psychiatric diagnosis based on Child and Adolescent Symptom Inventory-4R (Lavigne et al., 2009) for control children; and (4) Contraindications for MRI such as metallic implants or pregnancy. We used the WISC-IV General Ability Index (GAI) as a measure of Full Scale IQ. The GAI provides a comparable approximation of overall intellectual ability as represented by the WISC-IV Full-Scale IQ score, yet is less sensitive to the influence of working memory and processing speed (Prifitera et al., 1998; Weiss et al., 1999; Saklofske et al., 2004). For participants with WASI scores, we used the Tellegen and Briggs (1967) formula to convert WASI subtest scores into WISC-IV Index scores. In addition, we collected the ADHD Rating Scale: Home Version from parents (DuPaul et al., 1998). Five children in the ASD group were on stimulants that were withheld for at least $24 \mathrm{~h}$ before scanning; in addition one child with ASD was on non-stimulant and anti-anxiety medications that could not be withdrawn. All remaining children were not medicated.

\section{IMAGING PROTOCOL}

Echo-planar images were acquired on a Siemens Trio $3 \mathrm{~T}$ with parameters: $3 \mathrm{~mm}$ isotropic resolution $(3.0 \times 3.0 \times 2.5 \mathrm{~mm})$, $T R=2000 \mathrm{~ms}, T E=30 \mathrm{~ms}$, flip angle $=90^{\circ}, \mathrm{FOV}=192 \times$ $192 \mathrm{~mm}$. Each child underwent two functional runs, a resting state run for 5:14 min in which children were asked to rest with eyes open and stay awake, followed by a task run during which children performed a sustained attention task modified from Zink et al. (2003). Children were instructed to focus on the center of the screen and press a button with their right hand for a triangle (target stimuli) among serially presented squares, circles, and rectangles, and to ignore anything else that may come up elsewhere on the screen. Each stimulus was presented for $750 \mathrm{~ms}$ within a $2000 \mathrm{~ms}$ interstimulus interval. Targets appeared on 25\% of the trials and the remaining trials were non-targets, requiring no motor response. Of these non-target trials, 25\% were presented with the central stimuli only and on the remaining trials, a distracter, a small flickering shape was flashed in the periphery in one of the four corners of the display. On half of these distracter trials, the flickering shape was an open circle, whereas on the remaining half of the distracter trials, the shape was variable (e.g., star, diamond) and colorful. Therefore, the breakdown of the types of trials was 25\% target, $25 \%$ non-target without distracter, 25\% non-target with familiar distracter, and 25\% nontarget with novel distracter. The task consisted of 168 total trials presented in an event-related design with appropriate jitter determined by Optseq2 (http://surfer.nmr.mgh.harvard.edu/optseq/) and lasted 5:46 min. Trial types are not pertinent to the present results as they were regressed out from the connectivity analysis, and therefore, the only difference in connectivity between the resting and task runs was driven by the attentional state of the subject, unconstrained in the resting run and sustained in the task run. Structural images were also acquired for each subject, with a high resolution sagittal T1-weighted structural scan using a 3D MPRAGE sequence with a scan time of 8:05 min and the following parameters: $T R=2530 \mathrm{~ms}, T E=3.5 \mathrm{~ms}, 256 \times 256-\mathrm{mm}$ FOV, 176-mm slab with 1 -mm-thick slices, and a $7^{\circ}$ flip angle. Head motion was minimized by foam cushions padding the space between the subject's head and the headcoil.

\section{IMAGE PREPROCESSING}

Images were processed in SPM8 (Wellcome Department of Cognitive Neurology, London, UK) using MATLAB (Version 7.1 Mathworks, Inc., Sherborn, MA) for both rest and task runs. The first four time points were excluded to allow for signal stabilization. Images were corrected for slice timing and translational and rotational motion by realigning to the first image of the session with INRIAlign (Freire et al., 2002). Images were then normalized to the SPM8 EPI template and resliced to $4 \mathrm{~mm}$ for computational efficiency, low pass filtered to exclude frequencies higher than $0.08 \mathrm{~Hz}$, followed by spatial smoothing with $4 \mathrm{~mm}$ FWHM. Contributions of motion and physiological noise to the time course of each voxel were removed by including the six motion parameters, signal from ventricle and white matter regions of interest with their respective first temporal derivatives, as regressors of no interest (Wise et al., 2004; Birn et al., 2006; Van Dijk et al., 2010). Further, constant offsets and linear trends were also removed. For the task run, an additional regressor of task conditions was included as being of no interest in order to prevent inflation of functional connectivity estimates by activation differences associated with task conditions (e.g., distracter present vs. absent trials; motor response vs. no motor response). If task conditions are not regressed out, even regions with no moment-to-moment correlations would appear functionally connected because subjects were responding to task conditions over the course of trials [see Jones et al. (2010) for discussion of this point]. Thus, this preprocessing step made the resting and task data comparable, differing only in the subjects' cognitive state [following Gordon et al. (2012a,b)]. The observed pattern of 
results did not change when task conditions were not regressed out (See Supplementary Materials).

To further restrict the effect of motion on functional connectivity estimates, volumes with greater than $0.5 \mathrm{~mm}$ framewise displacement (FD) or temporal derivative of timecourses-root mean square variance over voxel (DVARS) greater than.5\% of the whole brain mode value were excluded (as recommended by Power et al. (2012). This "scrubbing" procedure retained 120 timepoints $(4 \mathrm{~min})$ for each child for further analysis. For retained volumes, mean FD did not differ between control (Rest: $M=0.158 \mathrm{~mm}, S D=0.061 \mathrm{~mm}$; Task: $M=0.167 \mathrm{~mm}, S D=$ $0.090 \mathrm{~mm}$ ) and ASD (Rest: $M=0.171 \mathrm{~mm}, S D=0.071 \mathrm{~mm}$; Task: $M=0.151 \mathrm{~mm}, S D=0.069 \mathrm{~mm})$ children during rest $(p=$ $0.58)$ or task $(p=0.57)$; further main effect of state $(p=0.63)$ and the group $\times$ state interaction was not significant $(p=$ 0.16 ) indicating that head micromovements did not depend on state. Further, the effects of any residual micromovements were removed by including Mean FD as a regressor in the second-level group analysis [following Satterthwaite et al. (2012)].

\section{LOCAL AND DISTANT CONNECTIVITY STRENGTH}

Following Sepulcre et al. (2010), the resulting smoothed images were used to map the local and distant functional connectivity. The time course of each voxel within a whole-brain mask excluding the cerebellum was correlated to every other voxel's time course, resulting in an $n \times n$ correlation matrix, where $\mathrm{n}$ is the dimension of the whole-brain mask $(n=33839)$. The correlation calculation is based on Pearson correlation coefficients $(r)$ and thresholded at $p=0.001$ FDR corrected at the individual level, to exclude less reliable pairwise connections [following Buckner et al. (2009)], resulting in a $\mathrm{r}$ threshold range of $0.32-0.34$ across individuals, after retaining only positive correlations. For each subject, a resting and task functional connectivity map was computed by averaging the r-to-Z Fisher transformed correlation values, for each voxel to voxels inside (for local connectivity map) and outside (for distant connectivity map) of a $14 \mathrm{~mm}$ radius. A $14 \mathrm{~mm}$ radius was chosen following Sepulcre et al. (2010) as they observed stable estimates of local connectivity for neighborhood radius values greater than $10 \mathrm{~mm}$ and no significant effect on distant connectivity estimates for radius more than $10-14 \mathrm{~mm}$. For discussion of the effects of neighborhood threshold, mask, smoothing kernels and $\mathrm{r}$ threshold see Buckner et al. (2009) and Sepulcre et al. (2010). We used connectivity degree weighted by strength (taking both the count of how many links connected to one voxel and their connectivity strength into account- see formulae in Supplementary Materials) as our connectivity estimate rather than connectivity degree alone as used by Sepulcre et al. (2010).

In order to identify regions where group differences in connectivity depended on cognitive state, we tested for Group (ASD, Control) X State (rest, task) interaction in second-level analysis. Subject-specific local and distant functional connectivity maps were entered into separate ANOVA models in SPM8 with Group and State as categorical variables and age and Mean FD as covariates of no interest. This analysis was thresholded at $p<0.05$ corrected for multiple comparisons based on Monte
Carlo simulation (Ward, 2000), which established the correction threshold at height $p<0.001, k=5$ voxels (for voxel size of $64 \mathrm{~mm}^{3}$ ). For clusters that survived the threshold, functional connectivity values were extracted using MarsBaR toolbox (Brett et al., 2002) from both resting and task runs and graphed to identify the nature of Group and State differences. Further, in regions showing Group $\times$ State interaction, we examined whether the magnitude of state-related functional connectivity change was related to inattention. For this analysis, a difference score was computed by subtracting the functional connectivity values from the Resting and Task runs and these difference scores were correlated with the inattention scores from the ADHD Rating Scale, separately for ASD and control children.

To visualize the change in distant functional connectivity patterns from resting to task states, we conducted a seed-based connectivity analysis using regions showing Group $\times$ Task interaction as seeds. For each subject at each state, the average timecourse of each significant seed cluster was extracted using MarsBaR and correlated with the timecourse of all other voxels in the brain; $r$ values were converted to $Z$ using Fisher's transformation. During the correlation calculation, we also regressed out signals of no interest, including timecourses from ventricle, white matter and six motion parameters with their respective first temporal derivatives. Then an averaged group map for each state was generated and visualized (at a range of thresholds $0.1-0.4$ ) on the cortical surface using the population-average, landmarkand surface-based (PALS) surface and plotted using Caret software (Van Essen, 2005). These results are depicted in Figures 1-4. This analysis allowed us to see the nature of change in the pattern of distant connectivity across states.

\section{GLOBAL GRAPH THEORY MEASURES}

We calculated two measures of network topology on a voxel-level graph, global efficiency and modularity, using the brain connectivity toolbox created by Sporns and colleagues (https://sites. google.com/site/bctnet/measures/list); the images were downsampled to $6 \mathrm{~mm}$ voxel size for computational efficiency [see Rubinov and Sporns (2010) and formulae in Supplementary Materials]. These graph measures were calculated by generating the undirected binary whole brain graph (excluding cerebellum as mentioned before), through thresholding the $9736 \times 9736$ correlation matrix (each $6 \mathrm{~mm}^{3}$ voxel to every other voxel) with the same FDR-corrected $\mathrm{r}$ threshold used for calculating local and distant connectivity. We also examined the effect of lower $r$ thresholds (0.2, 0.1 respectively) on the two graph measures (see Supplementary Materials) to show that our findings were not biased by more stringent $r$ threshold selection. For each subject, global efficiency and modularity were calculated for both the resting and task runs and entered into separate ANOVA models in $\mathrm{R}$ (http://cran.r-project.org) with Group and State as categorical variables with age and mean FD as covariates of no interest similar to the local/distant connectivity analysis above. Similarly, we also examined whether the magnitude of state-related change in global efficiency and modularity (Task-Resting difference) correlated with the inattention score of the ADHD Rating Scale, separately in the two groups. 


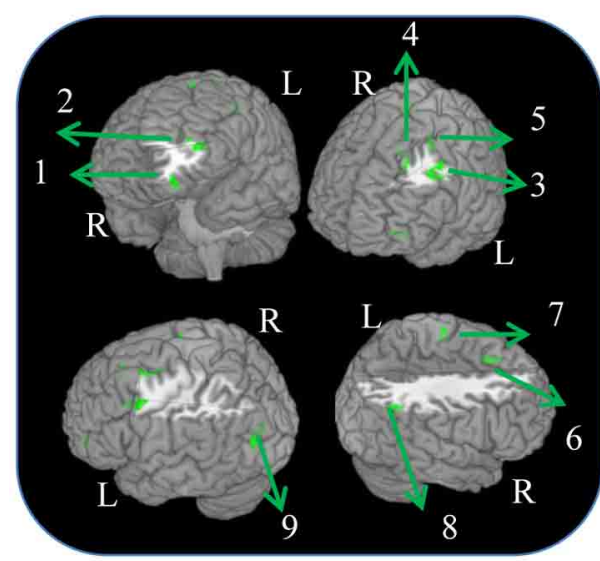

Orbital Frontal Gyrus (BA 11)

Group

$\rightarrow$ ASD

$\rightarrow$ CONTROL
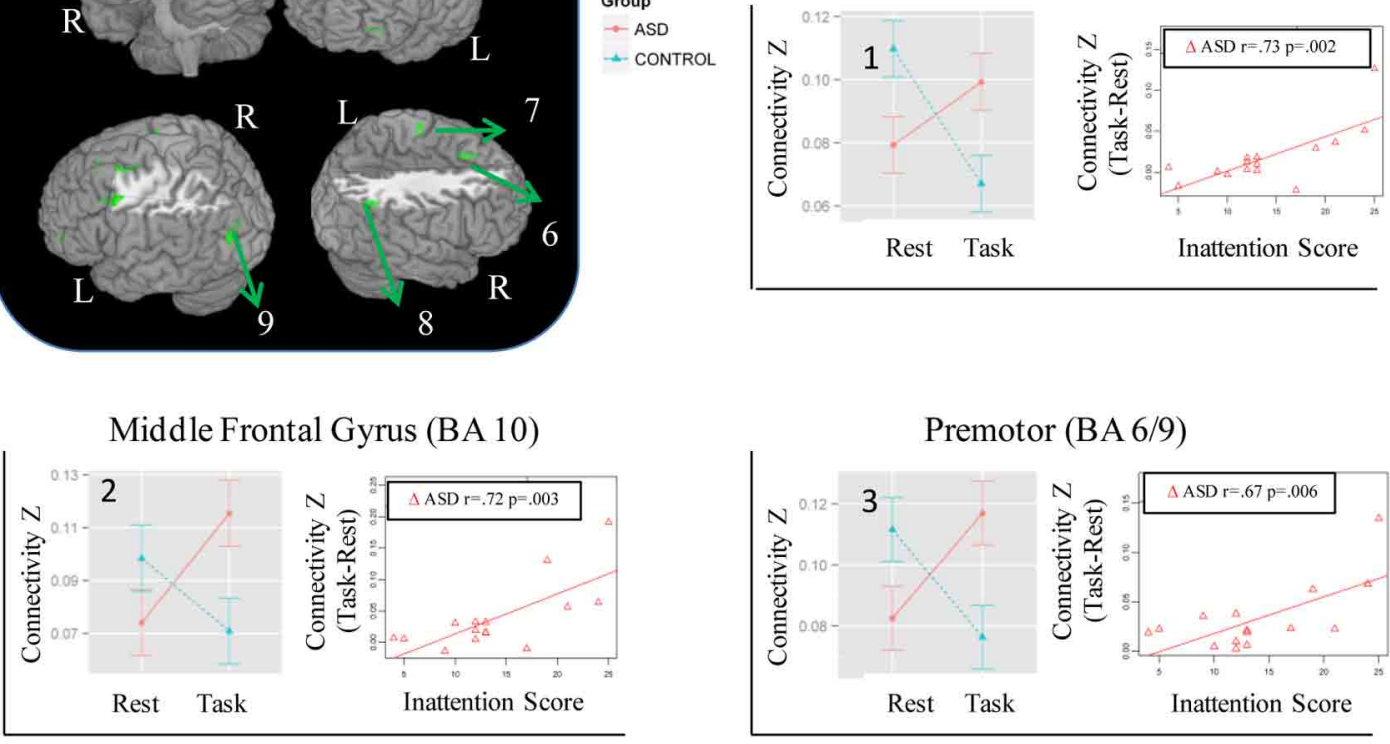

Premotor (BA 8)

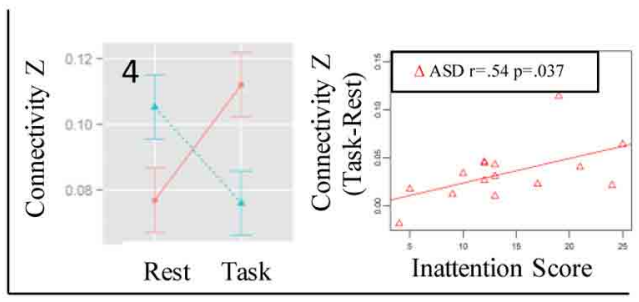

SMA (BA 32/8)

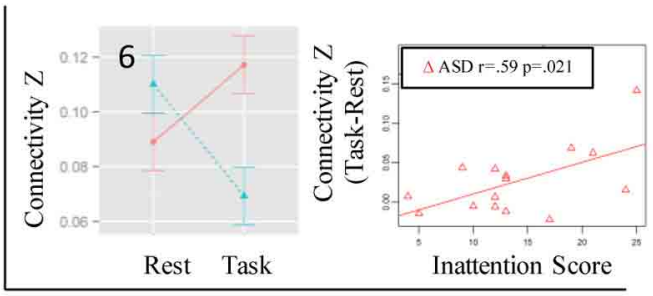

Angular Gyrus (BA 39/40)

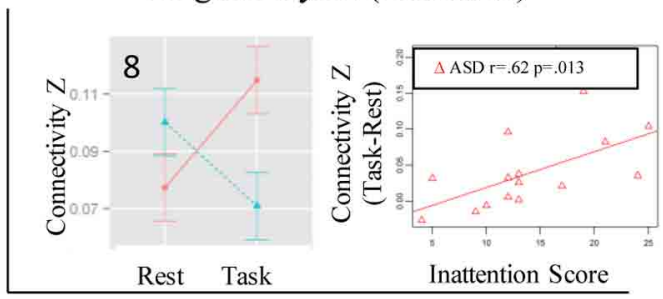

Premotor (BA 6/9)

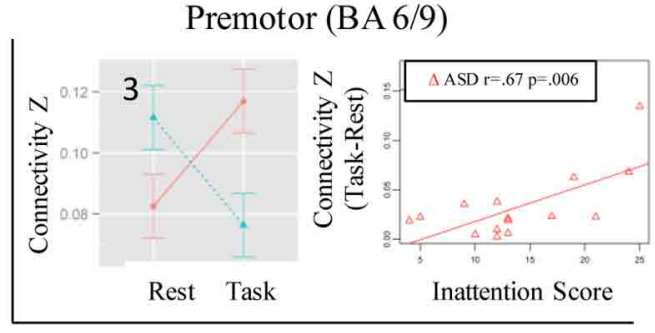

Premotor (BA 6)

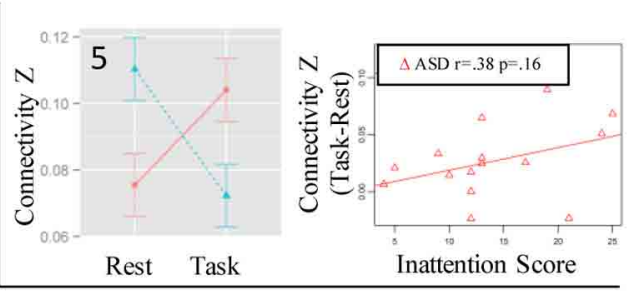

Paracentral Lobule (BA 6)

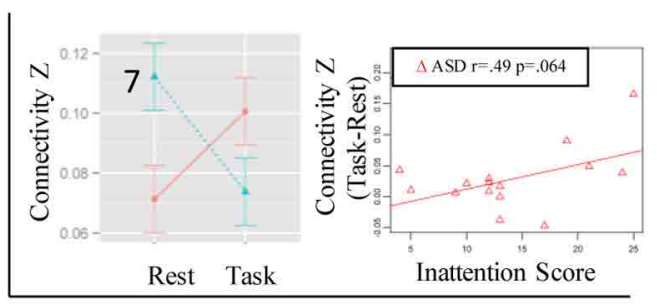

Posterior MTG (BA 19/39)

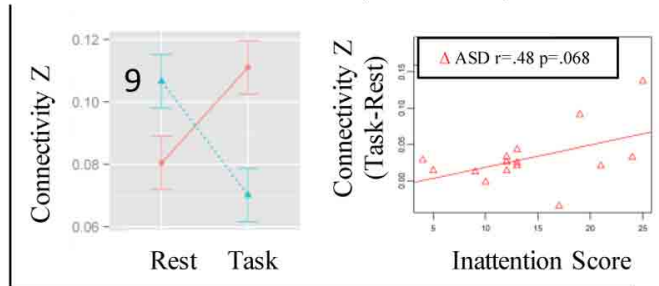

FIGURE 1 | Regions showing Group $\times$ State interaction for distant connectivity. Each region is identified with a number on the brain image in the top left corner. The corresponding graphs showing the interaction and correlation with inattention scores in the ASD group are identified with the same number. 


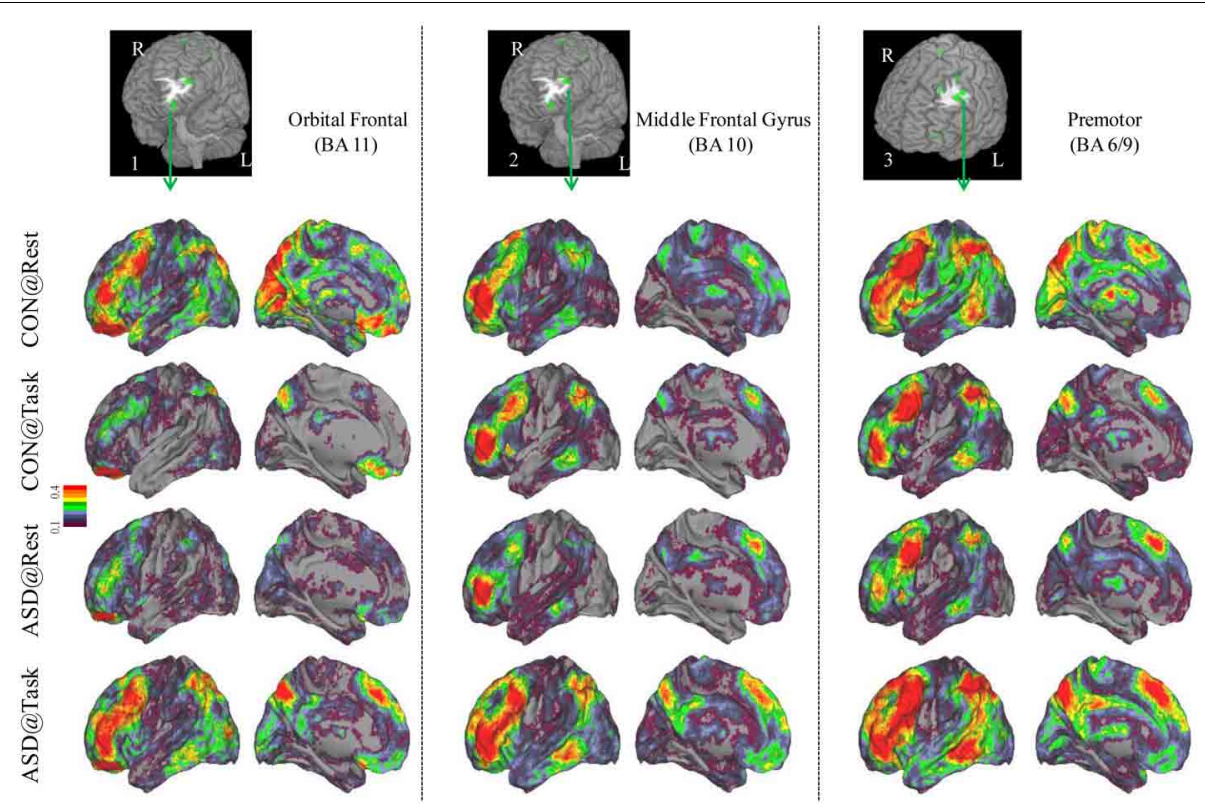

FIGURE 2 | Seed-based connectivity maps of distant functional connectivity patterns in resting and task states, for three clusters showing Group $\times$ Task interaction: left orbital frontal gyrus (BA 11) (left panel), left middle frontal gyrus (BA 10) (middle panel) and left premotor (BA 6/9) (right panel). Region numbers 1-3 on the left corner in the brain image correspond to the region number in Figure $\mathbf{1}$

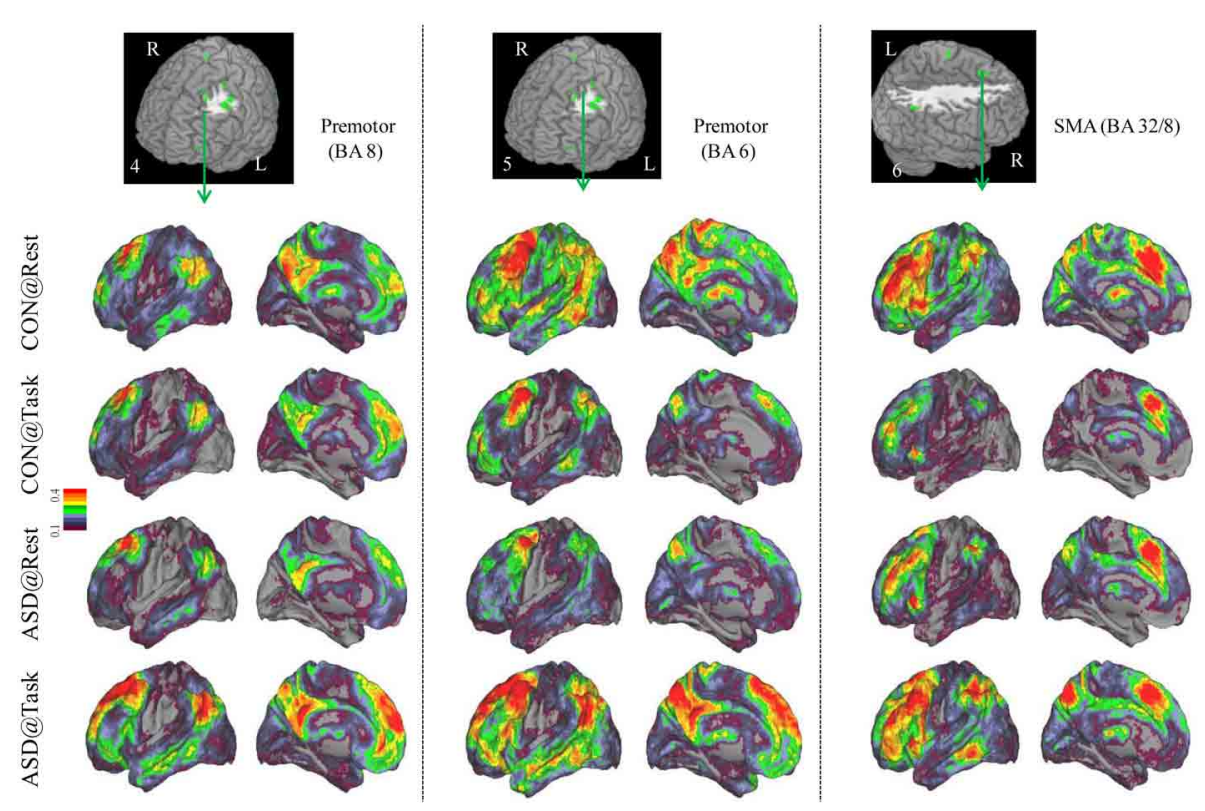

FIGURE 3 | Seed-based connectivity maps of distant functional connectivity patterns in resting and task states, for three clusters showing Group $\times$ Task interaction: left premotor (BA 8) (left panel), left premotor (BA 6) (middle panel) and SMA (BA 32/8) (right panel). Region numbers 4-6 on the left corner in the brain image correspond to the region number in Figure 1.

\section{RESULTS}

\section{BEHAVIOR}

For the task run, groups did not differ in target hits [ASD: $M=96.2 \%, \quad S D=8.0 \%$; Controls: $M=100 \%, S D=0 \%$, $t_{(14)}=1.9, p=0.08$ ] and false alarms [ASD: $M=0.08 \%$, $S D=0.3 \% ;$ Controls: $M=0.2 \%, \quad S D=0.5 \%, \quad t_{(25.7)}=1$, $p=0.33]$. However, target response was slower in ASD than control children [ASD: $M=602.1 \mathrm{~ms}, S D=77.6 \mathrm{~ms}$; Controls: $\quad M=513.2 \mathrm{~ms}, \quad S D=70.6 \mathrm{~ms}, \quad t_{(28.3)}=3.3$, $p=0.002]$. Mean ADHD Rating scores for Inattention $\left[t_{(21.7)}=5.4, \quad p<0.0001\right]$ and Hyperactivity-impulsivity $\left[t_{(21.4)}=3.9, p<0.001\right]$ were higher in ASD than control 


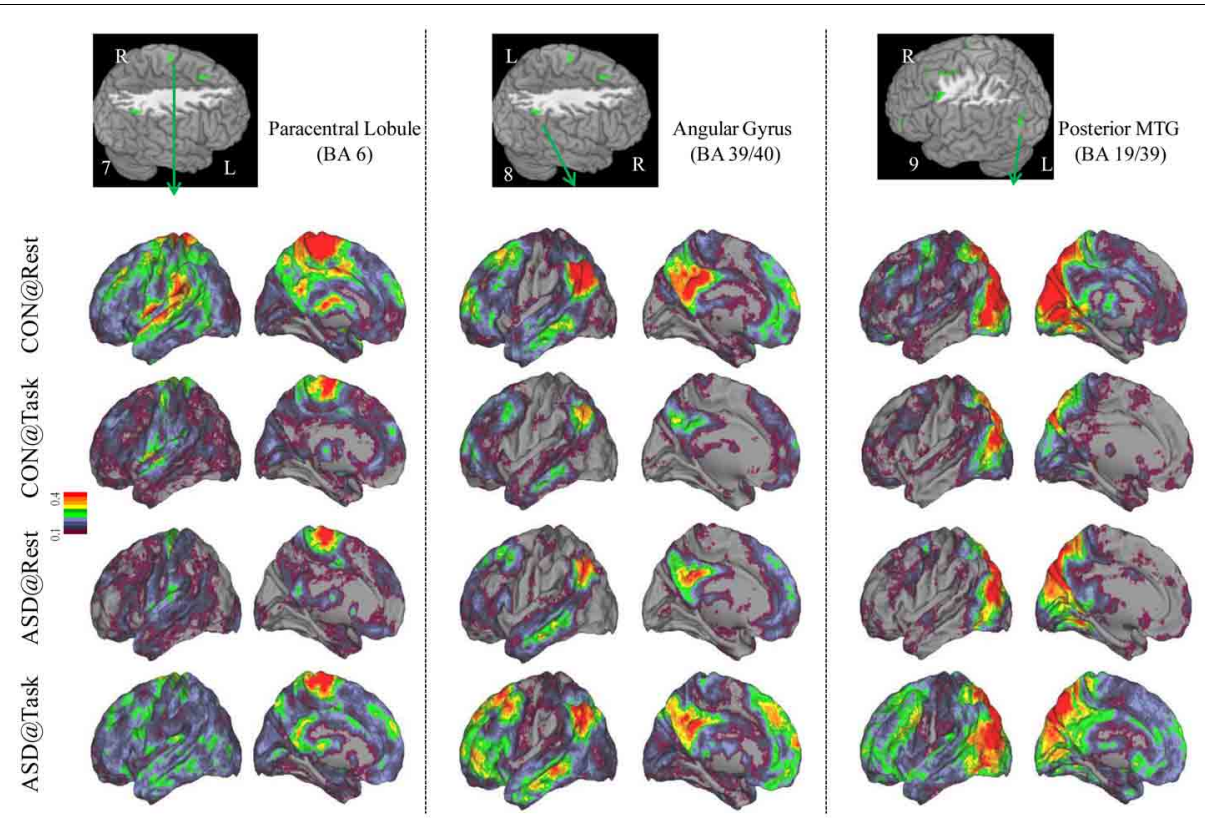

FIGURE 4 | Seed-based connectivity maps to understand distant functional connectivity patterns in resting and task states, for three clusters showing Group $x$ Task interaction: paracentral Lobule (BA 6) (left panel), right Angular Gyrus (BA 39/40) (middle panel) and Posterior MTG (BA 19/39) (right panel). Region numbers 7-9 on the left corner in the brain image correspond to the region number in Figure $\mathbf{1}$. children, indicating worse attentional function in ASD (see Table 1).

\section{LOCAL AND DISTANT FUNCTIONAL CONNECTIVITY}

While no regions showed a significant Group $\times$ State interaction for local connectivity, left frontal, right parietal, and left posterior temporal cortices showed the interaction in distant connectivity (Figure 1). In left frontal cortex, there were six clusters, a medial one including dorsal anterior cingulate extending into Supplementary Motor Area (SMA) (BA 32/8), and five lateral ones including dorsolateral prefrontal (middle frontal gyrus, BA 10; orbital gyrus, BA 11), and three in premotor cortex (BA 8, $6,6 / 9)$. In right parietal cortex, there were two clusters, a dorsomedial one in paracentral lobule (BA 6) and an inferior lateral one near angular gyrus (BA 39/40). The final posterior cluster was in left posterior middle temporal gyrus (BA 19/39). As can be seen in graphs in Figure 1 (cluster information in Table 2), in each of these regions, distant connectivity estimates were reduced in control children but increased in ASD children from resting to task state (See Table S1 for summary of mean, standard deviation and $p$-values in Supplementary Materials). Upon repeating the same analysis without regressing out trial conditions from the task run, similar Group $\times$ State interaction regions were found as above but with three exceptions - the paracentral lobule and BA 6 clusters did not survive the corrected threshold and the BA 6/9 cluster became larger (19 voxel vs. 16 voxel) (see Table S2 for summary of mean, standard deviation and $\mathrm{p}$ values in Supplementary Materials).

Seed-based connectivity maps for each of these regions showed that the connectivity map was more focal (i.e., smaller areas in the red-yellow intensity range) during the task relative to the resting
Table 2 | Regions showing Group (ASD, Control) x State (Resting, Sustained attention task) interaction for distant functional connectivity.

\begin{tabular}{|c|c|c|c|c|c|c|}
\hline & \multirow[t]{2}{*}{ Region } & \multicolumn{3}{|c|}{ MNI coordinates } & \multirow{2}{*}{$\begin{array}{l}\text { Cluster } \\
\text { size }\left(\mathrm{mm}^{3}\right)\end{array}$} & \multirow{2}{*}{$\begin{array}{l}\text { Peak } \\
Z \text {-score }\end{array}$} \\
\hline & & $x$ & $y$ & $z$ & & \\
\hline Left & $\begin{array}{l}\text { Orbital frontal } \\
\text { gyrus (BA 11) }\end{array}$ & -18 & 44 & -22 & 320 & 3.72 \\
\hline Left & $\begin{array}{l}\text { Middle frontal } \\
\text { gyrus (BA 10) }\end{array}$ & -42 & 48 & 6 & 384 & 3.82 \\
\hline Left & $\begin{array}{l}\text { Premotor (BA } \\
6 / 9 \text { ) }\end{array}$ & -42 & 12 & 38 & 1024 & 3.70 \\
\hline Left & Premotor (BA 8) & -26 & 20 & 50 & 512 & 4.26 \\
\hline Left & Premotor (BA 6) & -38 & 4 & 58 & 384 & 4.36 \\
\hline Medial & SMA (BA 32/8) & -2 & 24 & 46 & 384 & 3.57 \\
\hline Medial & $\begin{array}{l}\text { Paracentral } \\
\text { lobule (BA 6) }\end{array}$ & -2 & -20 & 70 & 320 & 3.34 \\
\hline Right & $\begin{array}{l}\text { Angular gyrus } \\
\text { (BA 39/40) }\end{array}$ & 50 & -60 & 38 & 320 & 4.66 \\
\hline Left & $\begin{array}{l}\text { Posterior MTG } \\
\text { (BA 19/39) }\end{array}$ & -46 & -80 & 18 & 512 & 3.87 \\
\hline
\end{tabular}

run, for the control group. In contrast, for the ASD group, the connectivity map was more diffuse (i.e., larger areas in the redorange intensity range) during the task relative to the resting run (See Figures 2-4); Figures showing difference maps ( $t$-test $p<$ $0.005,5$ voxels) comparing groups at each state (Figures S1-S3) and states for each group (Figures S4-S6) are in Supplementary Materials. 


\section{GLOBAL GRAPH THEORY MEASURES}

Group $\times$ State interaction was observed in global efficiency $\left[F_{(1,29)}=7.78, p=.009\right]$; post-hoc $t$-tests showed that global efficiency decreased from resting to the task run in control children $\left[t_{(15)}=2.72, p=0.016\right]$ but did not change significantly in ASD children $\left[t_{(14)}=0.96, p=0.36\right.$ ] (See bar graph in Figure 5). Further, the groups did not differ significantly in global efficiency during the resting $\left[t_{(22,7)}=1.3, p=0.21\right]$ or task $\left[t_{(26,7)}=\right.$ $1.22, p=0.23$ ] runs.

Modularity also showed a Group $\times$ State interaction $\left[F_{(1,29)}=9.45, p=0.005\right]$; post-hoc $t$-tests showed that modularity decreased in ASD children $\left[t_{(14)}=2.62, p=0.02\right]$ but did not change significantly in control children $\left[t_{(15)}=1.5, p=\right.$ 0.15] (See bar graph in Figure 5). Further, ASD children had higher modularity than controls $\left[t_{(23.5)}=2.31, p=0.03\right]$ during the resting run, but the groups did not differ during the task run $\left[t_{(28.8)}=0.85, p=0.40\right]$. These observed patterns did not change when task conditions were not regressed out (See Table S3).

\section{CORRELATION OF STATE-RELATED CHANGE IN DISTANT CONNECTIVITY WITH INATTENTION SCORES}

The magnitude of increase in distant connectivity from resting to the task state in clusters showing Group $\times$ State interaction correlated positively with the inattention scores in ASD children, indicating that those with greater attention problems in everyday life showed a stronger increase in distant connectivity from resting to the task run (see scatterplots in Figure 1). Specifically, correlation was significant in dorsolateral prefrontal (BA 11: $r=0.73, p=0.002$; BA 10: $r=0.72, p=0.003$ ), premotor (BA 8: $r=0.54, p=0.037$; BA 6/9: $r=0.67, p=0.006$ ), supplementary motor (BA 32/8: $r=0.59, p=0.021$ ), and in right angular gyrus (BA 39/40: $r=0.62, p=0.013$ ). In the remaining three clusters, premotor (BA $6, r=0.38, p=0.16$ ), paracentral lobule $(r=0.49, p=0.064)$, and middle temporal $(r=0.48, p=0.068)$, the correlation did not reach significance. The amount of task-related increase of global efficiency $(r=0.53$, $p=0.044)$ and decrease of modularity $(r=-0.67, p=0.007)$ in ASD children also correlated with inattention scores (see scatterplots in Figure 5). Correlations were not significant in control children $(p s>0.077)$, for either regions showing Group $\times$ State interaction or graph theory measures.

\section{DISCUSSION}

We used a voxel-wise method to characterize local and distant functional connectivity in two cognitive states, resting and sustained attention, in pre-adolescent children with ASD and control children. Results showed that state-related changes in distant functional connectivity differed between groups in prefrontal, premotor, parietal, and posterior temporal cortical regions known to be associated with cognitive control and spatial attention. In these regions, distant connectivity, defined by the weighted strength of each voxel's temporal correlation with all voxels in the brain outside of its local neighborhood, increased in ASD children but reduced in control children, during sustained attention relative to a preceding resting state. Seed-based maps further confirmed that as hypothesized, reduced distant connectivity in control children reflected a more focal network topology during task than during the resting state. In contrast, contrary to our hypothesis, ASD children showed increased distant connectivity, reflected in a more diffuse network topology, during task than during the resting state. The magnitude of staterelated increase in distant connectivity of prefrontal, premotor, and lateral parietal regions correlated positively with ASD children's inattention as measured by parent report on the ADHD Rating Scale. The resting versus task state comparison represents a distinction between attention that is unconstrained relative to

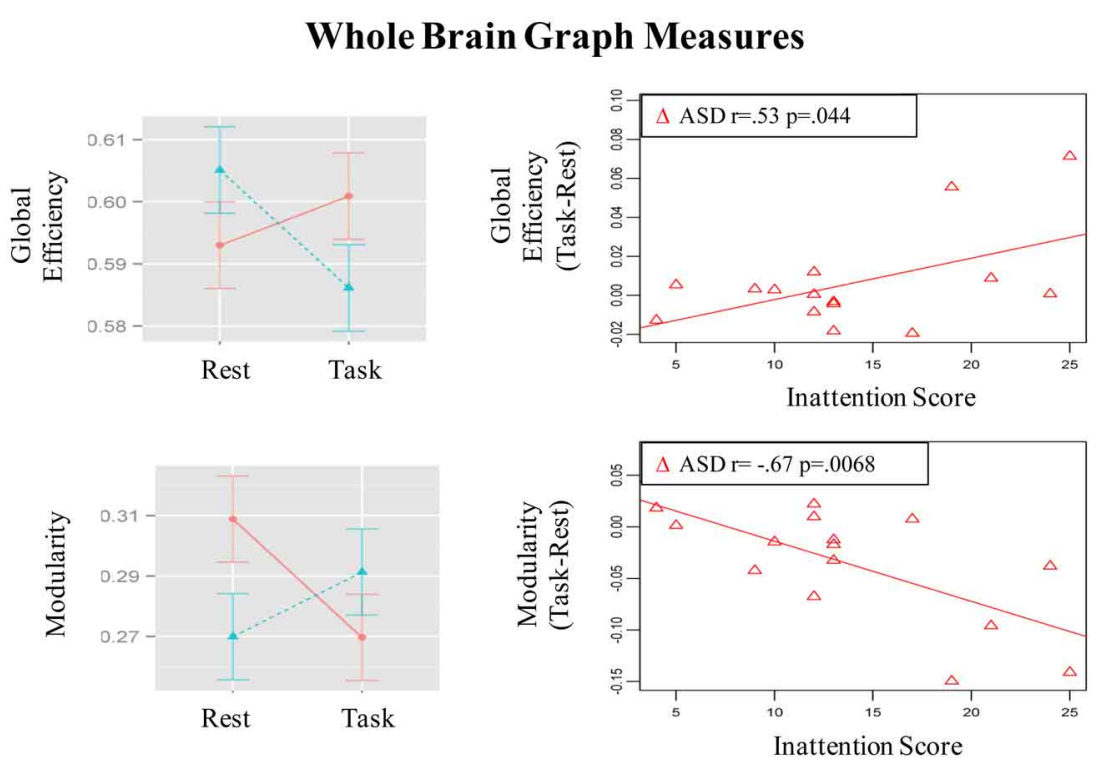

FIGURE 5 | Graphs depicting Group x State interaction for graph theory measures and correlation of the magnitude of state-related change (Task-Rest) with inattention scores in the ASD group. 
that which is constrained by task-goals (e.g., monitoring for a target shape), respectively. As ASD children transition between the unconstrained state to a sustained attention demand, functional connectivity of frontal and parietal regions becomes more widespread, a property that may be maladaptive as it predicted greater attention problems in everyday life.

Some methodological considerations are important to note for interpreting the observed results. First, distant connectivity maps represent moderately high positive correlations $(\sim 0.33)$ between voxels. Further, global signal regression was not performed and therefore, positive/negative correlation value distributions were not altered during preprocessing (Murphy et al., 2009). Thus, interpretation of the observed results is limited to state-related changes in positive functional connectivity. Second, head motion was addressed using "scrubbing" procedures recommended by Power et al. (2012), resulting in retaining $4 \mathrm{~min}$ of data in each run for each child. While longer durations are desirable, $4 \mathrm{~min}$ is adequate to yield reliable connectivity estimates (Van Dijk et al., 2010). Residual motion was further addressed by using mean FD as a regressor in second-level analysis. As the number of volumes removed and mean FD did not differ between groups, the observed results cannot be attributed to differences in head motion. Third, the sustained attention task included manipulation of distracting information. As our primary aim was to examine effects of cognitive state, task conditions were regressed out, in order to ensure that group differences in connectivity were not driven by differential response to distraction. Importantly, repeating the analysis without regressing out task conditions resulted in a similar pattern of state-related group differences (Table S2), suggesting that the observed group differences were not driven by manipulation of task conditions. Fourth, scan order was fixed, with the resting state run acquired immediately before the task run. Order was not counterbalanced because pre-task and post-task resting state is not identical as task-related functional connectivity persists into the subsequent resting state, suggestive of a cognitive aftereffect (Gordon et al., 2012a). Fifth, our sample sizes of $15 / 16$ children per group are relatively small due to our design requiring two back-to-back $\mathrm{fMRI}$ runs satisfying strict motion criteria from the same child. Nonetheless, it is important to note that the small samples limit the generalizability of the observed results.

Distant but not local functional connectivity was sensitive to group differences in modulation by cognitive state. Efficient cortical processing is posited to reflect the balance of connectivity within local regions supported by U-fibers, and across disparate regions supported by long-range white matter tracts (Mesulam, 1998; Schmahmann et al., 2008). While both types of connectivity are present throughout cortex, regions differ in their dominant (e.g. local or distant) connectivity properties. Local hierarchical connections are more representative of sensory cortical areas whereas association cortices such as prefrontal, parietal, lateral temporal, and limbic/paralimbic, have more long-range distributed connections (Felleman and Van Essen, 1991; Mesulam, 1998). In a study with healthy adults, Sepulcre et al. (2010) showed that the local/distant processing topology was paralleled in voxel-wise functional connectivity of low-frequency BOLD signals such that visual and somatosensory cortices showed higher local connectivity whereas association cortices showed higher distant connectivity. Further, while performing a semantic classification task, local and distant connectivity patterns of regions relevant to that task changed relative to a resting state. Here, we found that any state-related changes in local connectivity did not differ between ASD and control children, at least at a threshold that corrected for multiple comparisons. The size of the local neighborhood, $14 \mathrm{~mm}$ sphere, was selected based upon Sepulcre et al.'s (2010) recommendation as being optimal for distinguishing regional topography. While that recommendation is based upon adult brain size, it applies to children of the ages examined here as normalization of pediatric brain images to adult stereotactic space has been validated in children as young as 7 years (Burgund et al., 2002; Kang et al., 2003). Lack of significant group differences in state-related modulation of local connectivity suggests that local processing as reflected in voxel-wise BOLD temporal correlations is typical in ASD, at least in the context of transitioning to a relatively easy sustained attention task state.

Distant connectivity was modulated atypically in ASD children during sustained attention relative to a resting state, specifically in regions associated with attentional function. These regions included left dorsolateral prefrontal cortex (BA 10, 11), dorsal anterior cingulate extending to SMA (BA 32/8), and lateral premotor regions (BA 6, 8, 6/9), which are often engaged during tasks requiring cognitive control (Bunge et al., 2002; Vaidya et al., 2005). In addition, there were two parietal clusters, in right paracentral lobule, perhaps associated with motor responses and right inferior parietal cortex associated with spatial attention (Shulman et al., 2010). Finally, there was a cluster in left posterior middle temporal cortex (BA 19/39), a region that children sometimes engage during cognitive control tasks (Rubia et al., 1999; Durston et al., 2003; Vaidya et al., 2005). In all these regions, distant connectivity during sustained attention reduced in control children but increased in ASD children, relative to a resting state. Seed-based connectivity of each of these regions disambiguated the rest-to-task connectivity changes by showing that control children had a focal or less extensive pattern of anterior-posterior connectivity networks during the task relative to resting state. In contrast, ASD children showed the opposite pattern, diffuse or more extensive connectivity networks during the task relative to resting state, suggestive of a lack of selective engagement of task-relevant networks. Such a failure ought to lead to worse performance, which was evident in slower target detection speed in ASD children, while maintaining high accuracy. Further, the extent of increased distant connectivity from rest-to-task states in cognitive control (e.g., prefrontal, medial frontal, premotor) and spatial attention (e.g., lateral parietal) regions was associated with attention problems in everyday behavior as ASD children with larger increases in connectivity had worse inattention scores on the ADHD Rating Scale. Diffuse network engagement during an attentionally demanding state in ASD children may relate to the putative imbalance of inhibitory to excitatory connections associated with glutamatergic (Bejjani et al., 2012) and/or GABAergic dysfuntion (Rojas et al., 2013). If indeed so, then our results suggest that 
the inhibitory/excitatory milieu of the brain in ASD is modulated by cognitive state in a manner that differs from typical development. Whatever the physiological basis, it appears that in transitioning from a resting to sustained attention state, ASD children exhibited indiscriminate cortical network engagement, which may underlie their functional impairment in the domain of attention.

Group differences in state-related distant connectivity changes were apparent in two graph theory metrics, modularity and global efficiency, which quantify properties of global network organization (Rubinov and Sporns, 2010). Modularity describes the extent to which a network is organized into densely connected modules that are segregated from each other and global efficiency describes the average number of connections to be crossed to go from each voxel to every other voxel in the brain. In control children, global efficiency reduced during sustained attention compared to a resting state; this reduction reflects increased path length, which is consistent with a less extensive network observed during task relative to the resting state. This metric did not show significant difference across states in ASD children. ASD children's modularity reduced during task relative to the resting state, a pattern suggesting increased noise between modules (Bullmore and Sporns, 2009; Rubinov and Sporns, 2010), which is consistent with the observation of a more extensively connected network in ASD children during task than resting state. Even though state-related change was significant only for modularity in ASD children, their amount of change in both graph theory measures predicted inattention scores. Further, comparison of the groups during the resting state showed results that were consistent with past studies using scalp-based imaging measures showing higher modularity [electroencephalography (Barttfeld et al., 2011; Boersma et al., 2013)] in ASD compared to control subjects. While lower global efficiency [magnetoencephalography (Tsiaras et al., 2011)] has been reported in ASD children, it did not differ significantly between groups in the present study. These findings add to the growing volume of studies showing that graph theory metrics are sensitive to inter-individual differences [e.g., age, neurological, and psychiatric disorder (Bullmore and Sporns, 2009)] as well as intra-individual differences [e.g., learning (Bassett et al., 2011), working memory performance (Stevens et al., 2012), IQ (Van den Heuvel et al., 2009)]. Establishing the sensitivity of such whole-brain network metrics to subject factors or cognitive state is an important step in assessing their potential for serving as biomarkers for psychiatric and developmental disorders.

The present findings contribute to developing theories of functional connectivity in ASD in four novel ways. First, they extend the notion that functional connectivity is abnormal in ASD to include transitions across cognitive states. Studies examining functional connectivity during task states that are highly demanding of attention (e.g., theory of mind, working memory, face processing) show reduced connectivity of task-selective networks comprising distant frontal-posterior regions in ASD (Just et al., 2012; Khan et al., 2013). It is plausible that a failure of task-selective engagement such as that suggested by more extensive voxel-wise distant connectivity networks observed here is paralleled in reduced functional connectivity of specific networks or regions. We are unable to effectively test this prediction within the present data because procedures for addressing head motion required excluding volumes with high head motion, making for sparse sampling of individual trial-types.

Second, our findings highlight that examination of highly comorbid deficits in ASD such as attentional function may be insightful about pathophysiology of ASD. Attentional dysfunction is a common comorbid condition in ASD, with over $40 \%$ of ASD children estimated to also meet criteria for attention deficit hyperactivity disorder (ADHD) (Leyfer et al., 2006; Yerys et al., 2009b; Sikora et al., 2012). We cannot formally diagnose ADHD in the present sample based solely on parental report on the ADHD Rating scale. However, average scores for inattention and hyperactivity/impulsivity were higher in ASD than control children and 6 of the 15 ASD children had clinically elevated scores for either Inattention or Hyperactivity/impulsivity, consistent with past reports (Yerys et al., 2009b; Rosenthal et al., 2013; Smithson et al., 2013). Attentional and executive dysfunction are common targets for intervention in ASD as they are associated with worse adaptive functioning (Gilotty et al., 2002; Sikora et al., 2012) and outcome in adulthood [see review Hume et al. (2009)]. To the extent that some level of attentional dysfunction always accompanies ASD, it is important to characterize the underlying neural signatures, especially if they prove to be unique to ASD. Thus, it would be important to conduct a similar study in children with ADHD to specify the extent to which our results reflect a general or disorder-specific correlate of transitioning between attentional states.

Third, our graph theory findings contribute to the growing body of studies of large-scale network structure of the brain by showing that modularity and global efficiency were sensitive to ASD and to manipulation of cognitive state. Demonstrating such sensitivity contributes to the potential of such connectivity metrics to serve as biomarkers for psychiatric and developmental disorders. Fourth, the present results highlight the importance of considering cognitive state in current theories of functional connectivity in ASD. It is likely that neither under- nor overconnectivity may characterize ASD in absolute terms but that the nature of alteration may depend upon the specific cognitive state. Mixed findings across task-evoked functional connectivity studies may reflect nuanced differences in the subjects cognitive state induced not just by experimental demands but also the individual's experience of the task as high/low arousing, easy/hard, boring/enjoyable. Furthermore, specific networks may be more susceptible to cognitive state differences than others. As this area of investigation evolves, consideration of task demands, networks, and individual subject characteristics ought to be productive in resolving the status of connectivity abnormality in ASD.

\section{ACKNOWLEDGMENTS}

We thank Evan Gordon for helpful discussion of methods. This work was funded by MH084961 from NIMH to Chandan J. Vaidya, and supported by the Intellectual and Developmental Disabilities Research Center, Children's National Medical Center Grant [HD040677-07]. 


\section{SUPPLEMENTARY MATERIAL}

The Supplementary Material for this article can be found online at: http://www.frontiersin.org/Human_Neuroscience/ 10.3389/fnhum.2013.00482/abstract

FIGURE S1 | Group differences in seed-based connectivity maps in resting and task states, for three clusters showing Group $\times$ Task interaction: left orbital frontal gyrus (BA 11) (left panel), left middle frontal gyrus (BA 10) (middle panel) and left premotor (BA 6/9) (right panel). Region numbers 1-3 on the left corner in the brain image correspond to the region number in Figure 1

FIGURE S2 | Group differences in seed-based connectivity maps in resting and task states, for three clusters showing Group $\times$ Task interaction: left premotor (BA 8) (left panel), left premotor (BA 6) (middle panel) and SMA (BA 32/8) (right panel). Region numbers 4-6 on the left corner in the brain image correspond to the region number in Figure 1.

FIGURE S3 | Group differences in seed-based connectivity maps in resting and task states, for three clusters showing Group $\times$ Task interaction:

Paracentral Lobule (BA 6) (left panel), right Angular Gyrus (BA 39/40)

(middle panel) and Posterior MTG (BA 19/39) (right panel). Region numbers 7-9 on the left corner in the brain image correspond to the region number in Figure 1

FIGURE S4 | State differences in seed-based connectivity maps within each group, for three clusters showing Group $\times$ Task interaction: left orbital frontal gyrus (BA 11) (left panel), left middle frontal gyrus (BA 10) (middle panel) and left premotor (BA 6/9) (right panel). Region numbers 1-3 on the left corner in the brain image correspond to the region number in Figure 1

FIGURE S5 | State differences in seed-based connectivity maps within each group, for three clusters showing Group $\times$ Task interaction: left premotor (BA 8) (left panel), left premotor (BA 6) (middle panel) and SMA (BA 32/8) (right panel). Region numbers 4-6 on the left corner in the brain image correspond to the region number in Figure $\mathbf{1}$

FIGURE S6 | State differences in seed-based connectivity maps within each group, for three clusters showing Group $\times$ Task interaction: Paracentral Lobule (BA 6) (left panel), right Angular Gyrus (BA 39/40) (middle panel) and Posterior MTG (BA 19/39) (right panel). Region numbers 7-9 on the left corner in the brain image correspond to the region number in Figure 1.

Rev. Neurosci. 10, 186-198. doi: $10.1038 / \mathrm{nrn} 2575$

Bunge, S. A., Dudukovic, N. M., Thomason, M. E., Vaidya, C. J., and Gabrieli, J. D. E. (2002). Immature frontal lobe contributions to cognitive control in children: evidence from fMRI. Neuron 33, 301-311. doi: $\quad 10.1016 /$ S0896-6273(01) 00583-9

Burgund, E. D., Kang, H. C., Kelly, J. E., Buckner, R. L., Snyder, A. Z., Petersen, S. E., et al. (2002). The feasibility of a common stereotactic space for children and adults in fMRI studies of development. Neuroimage 17, 184-200. doi: 10.1006/nimg.2002.1174

Cherkassky, V. L., Kana, R. K., Keller, T. A., and Just, M. A. (2006). Functional connectivity in a baseline resting-state network in autism. Neuroreport 17, 1687-1690. doi: 10.1097/01.wnr.0000239956. 45448.4c

Di Martino, A., Kelly, C., Grzadzinski, R., Zuo, X.-N., Mennes, M., Mairena, M. A., et al. (2011). Aberrant striatal functional connectivity in children with autism. Biol. Psychiatry 69, 847-856. doi: 10.1016/j.biopsych.2010.10.029

DuPaul, G. J., Power, T. J., Anastopoulos, A. D., and Reid, R. (1998). ADHD Rating Scale-IV: Checklists, Norms, and Clinical Interpretation. (New York, NY: Guilford Press).

Durston, S., Tottenham, N. T., Thomas, K. M., Davidson, M. C., Eigsti, I.-M., Yang, Y., et al. (2003). Differential patterns of striatal activation in young children with and without ADHD. Biol. Psychiatry 53,
871-878. doi: 10.1016/S0006-3223 (02)01904-2

Felleman, D. J., and Van Essen, D. C. (1991). Distributed hierarchical processing in the primate cerebral cortex. Cereb. Cortex 1, 1-47. doi: 10.1093/cercor/1.1.1

Fox, M. D., Snyder, A. Z., Zacks, J. M., and Raichle, M. E. (2006). Coherent spontaneous activity accounts for trial-to-trial variability in human evoked brain responses. Nat. Neurosci. 9, 23-25. doi: $10.1038 / \mathrm{nn} 1616$

Fransson, P. (2006). How default is the default mode of brain function? Further evidence from intrinsic BOLD signal fluctuations. Neuropsychologia 44, 2836-2845. doi: 10.1016/ j.neuropsychologia.2006.06.017

Freire, L., Roche, A., and Mangin, J.-F. (2002). What is the best similarity measure for motion correction in fMRI time series. IEEE Trans. Med. Imaging 21, 470-484. doi: 10.1109/TMI.2002.1009383

Fukunaga, M., Horovitz, S. G., van Gelderen, P., de Zwart, J. A., Jansma, J. M., Ikonomidou, V. N. et al. (2006). Large-amplitude, spatially correlated fluctuations in BOLD fMRI signals during extended rest and early sleep stages. Magn. Reson. Imaging 24, 979-992. doi: 10.1016/j.mri. 2006.04.018

Gilotty, L., Kenworthy, L., Sirian, L., Black, D. O., and Wagner, A. E. (2002). Adaptive skills and executive function in autism spectrum disorders. Child Neuropsychol. 8, 241-248. doi: 10.1076/chin.8.4.241.13504 
Gordon, E. M., Breeden, A. L., Bean, S. E., and Vaidya, C. J. (2012a). Working memory-related changes in functional connectivity persist beyond task disengagement. Hum. Brain Mapp. doi: 10.1002/hbm. 22230. [Epub ahead of print].

Gordon, E. M., Stollstorff, M., Devaney, J. M., Bean, S., and Vaidya, C. J. (2012b). Effect of dopamine transporter genotype on intrinsic functional connectivity depends on cognitive state. Cereb. Cortex 22, 2182-2196. doi: 10.1093/cercor/bhr305

Gordon, E. M., Stollstorff, M., and Vaidya, C. J. (2012c). Using spatial multiple regression to identify intrinsic connectivity networks involved in working memory performance. Hum. Brain Mapp. 33, 1536-1552. doi: 10.1002/hbm.21306

Gotts, S. J., Simmons, W. K., Milbury, L. A., Wallace, G. L., Cox, R. W., and Martin, A. (2012). Fractionation of social brain circuits in autism spectrum disorders. Brain 135, 2711-2725. doi: 10.1093/brain/aws160

Greicius, M. D., Kiviniemi, V., Tervonen, O., Vainionpää, V., Alahuhta, S., Reiss, A. L., et al. (2008). Persistent default-mode network connectivity during light sedation. Hum. Brain Mapp. 29, 839-847. doi: 10.1002/hbm.20537

Hampson, M., Olson, I. R., Leung, H.-C., Skudlarski, P., and Gore, J. C. (2004). Changes in functional connectivity of human MT/V5 with visual motion input. Neuroreport 15, 1315-1319. doi: 10.1097/01.wnr.0000129997. 95055.15

Heine, L., Soddu, A., Gómez, F., Vanhaudenhuyse, A., Tshibanda, L., Thonnard, M., et al. (2012). Resting state networks and consciousness: alterations of multiple resting state network connectivity in physiological, pharmacological, and pathological consciousness States. Front. Psychol. 3:295. doi: 10.3389/fpsyg.2012.00295

Hill, E. L. (2004). Executive dysfunction in autism. Trends Cogn. Sci. 8, 26-32. doi: 10.1016/j.tics.2003.11.003

Hume, K., Loftin, R., and Lantz, J. (2009). Increasing independence in autism spectrum disorders: a review of three focused interventions. J. Autism Dev. Disord. 39, 1329-1338. doi: 10.1007/s10803-009-0751-2

Jiang, T., He, Y., Zang, Y., and Weng, X. (2004). Modulation of functional connectivity during the resting state and the motor task. Hum. Brain Mapp. 22, 63-71. doi: 10.1002/hbm.20012

Jones, T. B., Bandettini, P. A., Kenworthy, L., Case, L. K., Milleville, S. C., Martin, A., et al. (2010). Sources of group differences in functional connectivity: an investigation applied to autism spectrum disorder. Neuroimage 49, 401-414. doi: 10.1016/j.neuroimage.2009.07.051

Just, M. A., Keller, T. A., Malave, V. L., Kana, R. K., and Varma, S. (2012). Autism as a neural systems disorder: a theory of frontal-posterior underconnectivity. Neurosci. Biobehav. Rev. 36, 1292-1313. doi: 10.1016/j.neubiorev.2012.02.007

Kang, H. C., Burgund, E. D., Lugar, H. M., Petersen, S. E., and Schlaggar, B. L. (2003). Comparison of functional activation foci in children and adults using a common stereotactic space. Neuroimage 19, 16-28. doi: 10.1016/S1053-8119(03)00038-7

Kennedy, D. P., and Courchesne, E. (2008). The intrinsic functional organization of the brain is altered in autism. Neuroimage 39, 1877-1885. doi: 10.1016/j.neuroimage.2007.10.052

Kenworthy, L., Black, D. O., Harrison, B., Della Rosa, A., and Wallace, G. L. (2009). Are executive control functions related to autism symptoms in high-functioning children. Child Neuropsychol. 15, 425-440. doi: 10.1080/09297040 802646983

Kenworthy, L., Yerys, B. E., Anthony, L. G., and Wallace, G. L. (2008). Understanding executive control in autism spectrum disorders in the lab and in the real world. Neuropsychol. Rev. 18, 320-338. doi: 10.1007/s1 1065-008-9077-7

Kenworthy, L. E., Black, D. O., Wallace, G. L., Ahluvalia, T., Wagner, A. E., and Sirian, L. M. (2005). Disorganization: the forgotten executive dysfunction in high-functioning autism (HFA) spectrum disorders. Dev. Neuropsychol. 28, 809-827. doi: 10.1207/s15326942dn2803_4

Khan, S., Gramfort, A., Shetty, N. R., Kitzbichler, M. G., Ganesan, S., Moran, J. M., et al. (2013). Local and long-range functional connectivity is reduced in concert in autism spectrum disorders. Proc. Natl. Acad. Sci. U.S.A. 110, 3107-3112. doi: 10.1073/pnas. 1214533110

Lai, G., Pantazatos, S. P., Schneider, H., and Hirsch, J. (2012). Neural systems for speech and song in autism. Brain 135, 961-975. doi: 10.1093/brain/awr335
Lainhart, J. E., Bigler, E. D., Bocian, M., Coon, H., Dinh, E., Dawson, G., et al. (2006). Head circumference and height in autism: a study by the collaborative program of excellence in autism. Am. J. Med. Genet. A 140, 2257-2274. doi 10.1002/ajmg.a.31465

Latora, V., and Marchiori, M. (2001). Efficient behavior of small-world networks. Phys. Rev. Lett. 87, 198701. doi 10.1103/PhysRevLett.87.198701

Lavigne, J. V., Cromley, T., Sprafkin, J., and Gadow, K. D. (2009). The child and adolescent symptom inventoryprogress monitor: a brief diagnostic and statistical manual of mental disorders, 4th edition-referenced parent-report scale for children and adolescents. J. Child Adolesc. Psychopharmacol. 19, 241-252. doi: 10.1089/cap.2008.052

Leyfer, O. T., Folstein, S. E., Bacalman, S., Davis, N. O., Dinh, E., Morgan, J., et al. (2006). Comorbid psychiatric disorders in children with autism: interview development and rates of disorders. J. Autism Dev. Disord. 36, 849-861. doi: 10.1007/s10803-006-0123-0

Lopez, B. R., Lincoln, A. J., Ozonoff, S., and Lai, Z. (2005). Examining the relationship between executive functions and restricted, repetitive symptoms of Autistic Disorder. J. Autism Dev. Disord. 35, 445-460. doi: 10.1007/s10803-005-5035-x

Lord, C., Risi, S., Lambrecht, L., Cook, E. H., Leventhal, B. L., DiLavore, P. C., et al. (2000). The autism diagnostic observation schedulegeneric: a standard measure of social and communication deficits associated with the spectrum of autism. J. Autism Dev. Disord. 30, 205-223. doi: 10.1023/A:1005592401947

Lord, C., Rutter, M., and Le Couteur, A. (1994). Autism diagnostic interview-revised: a revised version of a diagnostic interview for caregivers of individuals with possible pervasive developmental disorders. J. Autism Dev. Disord. 24, 659-685. doi: 10.1007/BF02172145

Mennes, M., Kelly, C., Zuo, X.-N., Di Martino, A., Biswal, B., Castellanos, F. X., et al. (2010). Inter-individual differences in resting state functional connectivity predict task-induced BOLD activity. Neuroimage 50, 1690-1701. doi: 10.1016/j.neuroimage.2010.01.002

Mesulam, M. M. (1998). From sensation to cognition. Brain 121(Pt 6), 1013-1052. doi: 10.1093/brain/121.6.1013

Monk, C. S., Peltier, S. J., Wiggins, J. L., Weng, S.-J., Carrasco, M., Risi,
S., et al. (2009). Abnormalities of intrinsic functional connectivity in autism spectrum disorders. Neuroimage 47, 764-772. doi: 10.1016/j.neuroimage.2009.04.069

Müller, R.-A., Shih, P., Keehn, B., Deyoe, J. R., Leyden, K. M., and Shukla, D. K. (2011). Underconnected, but how. A survey of functional connectivity MRI studies in autism spectrum disorders. Cereb. Cortex 21, 2233-2243. doi: 10.1093/cercor/bhq296

Murphy, K., Birn, R. M., Handwerker, D. A., Jones, T. B., and Bandettini, P. A. (2009). The impact of global signal regression on resting state correlations: are anticorrelated networks introduced. Neuroimage 44, 893-905. doi: 10.1016/j.neuroimage.2008.09.036

Newman, M. E. J. (2006). Modularity and community structure in networks. Proc. Natl. Acad. Sci. U.S.A. 103, 8577-8582. doi: 10.1073/pnas.0601602103

Nir, Y., Hasson, U., Levy, I., Yeshurun, Y., and Malach, R. (2006). Widespread functional connectivity and fMRI fluctuations in human visual cortex in the absence of visual stimulation. Neuroimage 30, 1313-1324. doi: 10.1016/j.neuroimage.2005.11.018

Noonan, S. K., Haist, F., and Müller, R.-A. (2009). Aberrant functional connectivity in autism: evidence from low-frequency BOLD signal fluctuations. Brain Res. 1262, 48-63. doi: 10.1016/j.brainres.2008. 12.076

Power, J. D., Barnes, K. A., Snyder, A. Z., Schlaggar, B. L., and Petersen, S. E. (2012). Spurious but systematic correlations in functional connectivity MRI networks arise from subject motion. Neuroimage 59, 2142-2154. doi: 10.1016/j. neuroimage.2011.10.018

Prifitera, A., Weiss, L. G., and Saklofske, D. H. (1998). "The WISC-III in context," in WISC-III Clinical Use and Interpretation: ScientistPractitioner Perspectives, eds A. Prifitera and D. H. Saklofske (NewYork, NY: Academic Press), 1-38. doi: 10.1016/B978012564930-8/50002-4

Rojas, D. C., Singel, D., Steinmetz, S., Hepburn, S., and Brown, M. S. (2013). Decreased left perisylvian GABA concentration in children with autism and unaffected siblings. Neuroimage. doi: 10.1016/ j.neuroimage.2013.01.045. [Epub ahead of print].

Rosenthal, M., Wallace, G. L., Lawson, R., Wills, M. C., Dixon, E., Yerys, B. E., et al. (2013). Impairments 
in real-world executive function increase from childhood to adolescence in autism spectrum disorders. Neuropsychology 27, 13-18. doi: 10.1037/a0031299

Rubia, K., Overmeyer, S., Taylor, E., Brammer, M., Williams, S. C., Simmons, A., et al. (1999). Hypofrontality in attention deficit hyperactivity disorder during higher-order motor control: a study with functional MRI. Am. J. Psychiatry 156, 891-896.

Rubinov, M., and Sporns, O. (2010). Complex network measures of brain connectivity: uses and interpretations. Neuroimage 52, 1059-1069. doi: 10.1016/j.neuroimage.2009. 10.003

Saklofske, D. H., Prifitera, A., Weiss, L. G., Rolfhus, E., and Zhu, J. (2004). "Clinical Interpretation of the WISC-IV FSIQ and GAI," in WISCIV Clinical Use and Interpretation: Scientist-Practitioner Perspectives, (New York, NY: Academic Press), 36-71.

Satterthwaite, T. D., Wolf, D. H., Loughead, J., Ruparel, K., Elliott, M. A., Hakonarson, H., et al. (2012). Impact of in-scanner head motion on multiple measures of functional connectivity: relevance for studies of neurodevelopment in youth. Neuroimage 60, 623-632. doi: 10.1016/j.neuroimage.2011.12.063

Schmahmann, J. D., Smith, E. E., Eichler, F. S., and Filley, C. M. (2008). Cerebral white matter: neuroanatomy, clinical neurology, and neurobehavioral correlates. Ann. N.Y. Acad. Sci. 1142, 266-309. doi: 10.1196/annals.1444.017

Sepulcre, J., Liu, H., Talukdar, T., Martincorena, I., Yeo, B. T. T., and Buckner, R. L. (2010). The organization of local and distant functional connectivity in the human brain. PLoS Comput. Biol. 6:e1000808. doi: 10.1371/journal.pcbi.1000808

Shih, P., Keehn, B., Oram, J. K., Leyden, K. M., Keown, C. L., and Müller, R.-A. (2011). Functional differentiation of posterior superior temporal sulcus in autism: a functional connectivity magnetic resonance imaging study. Biol. Psychiatry 70, 270-277. doi: 10.1016/j.biopsych.2011.03.040

Shulman, G. L., Pope, D. L. W., Astafiev, S. V., McAvoy, M. P., Snyder, A. Z., and Corbetta, M. (2010). Right hemisphere dominance during spatial selective attention and target detection occurs outside the dorsal frontoparietal network. J. Neurosci. 30, 3640-3651. doi: 10.1523/JNEUROSCI.4085-09.2010

Sikora, D. M., Vora, P., Coury, D. L., and Rosenberg, D. (2012). Attentiondeficit/hyperactivity disorder symptoms, adaptive functioning, and quality of life in children with autism spectrum disorder. Pediatrics 130(Suppl. 2), S91-S97. doi: 10.1542/peds.2012-0900G

Smith, S. M., Fox, P. T., Miller, K. L., Glahn, D. C., Fox, P. M., Mackay, C. E., et al. (2009). Correspondence of the brain's functional architecture during activation and rest. Proc. Natl. Acad. Sci. U.S.A 106, 13040-13045. doi: 10.1073/pnas.0905267106

Smithson, P. E., Kenworthy, L., Wills, M. C., Jarrett, M., Atmore, K., and Yerys, B. E. (2013). Real world executive control impairments in preschoolers with autism spectrum disorders. J. Autism Dev. Disord. doi: 10.1007/s10803-012-1747-x. [Epub ahead of print].

Stevens, A. A., Tappon, S. C., Garg, A., and Fair, D. A. (2012). Functional brain network modularity captures inter- and intra-individual variation in working memory capacity. PLoS ONE 7:e30468. doi: 10.1371/journal.pone. 0030468

Tellegen, A., and Briggs, P. F. (1967). Old wine in new skins: grouping Wechsler subtests into new scales. J. Consult. Psychol. 31, 499-506. doi: 10.1037/h0024963

Tsiaras, V., Simos, P. G., Rezaie, R., Sheth, B. R., Garyfallidis, E., Castillo, E. M., et al. (2011). Extracting biomarkers of autism from MEG resting-state functional connectivity networks. Comput. Biol. Med. 41, 1166-1177. doi: 10.1016/j.compbiomed.2011.04.004

Vaidya, C. J., Bunge, S. A., Dudukovic, N. M., Zalecki, C. A., Elliott, G. R., and Gabrieli, J. D. E. (2005). Altered neural substrates of cognitive control in childhood ADHD: evidence from functional magnetic resonance imaging. Am. J. Psychiatry 162, 1605-1613. doi: 10.1176/appi.ajp.162.9.1605

Van den Heuvel, M. P., Stam, C. J., Kahn, R. S., and Hulshoff Pol, H. E. (2009). Efficiency of functional brain networks and intellectual performance. J. Neurosci. 29, 7619-7624. doi: 10.1523/JNEUROSCI.1443-09.2009
Van Dijk, K. R. A., Hedden, T., Venkataraman, A., Evans, K. C., Lazar, S. W., and Buckner R. L. (2010). Intrinsic functional connectivity as a tool for human connectomics: theory, properties, and optimization. J. Neurophysiol. 103, 297-321. doi: 10.1152/jn.00783.2009

Van Essen, D. C. (2005). A population-average, landmarkand surface-based (PALS) atlas of human cerebral cortex. Neuroimage 28, 635-662. doi: 10.1016/j.neuroimage.2005.06.058

Vincent, J. L., Patel, G. H., Fox, M. D., Snyder, A. Z., Baker, J. T., Van Essen, D. C., et al. (2007). Intrinsic functional architecture in the anaesthetized monkey brain. Nature 447 , 83-86. doi: 10.1038/nature05758

Von dem Hagen, E. A. H., Stoyanova, R. S., Baron-Cohen, S., and Calder, A. J. (2012). Reduced Functional Connectivity within and Between "social" Resting State Networks in Autism Spectrum Conditions. Social Cognitive and Affective Neuroscience. Available online at: http://www.ncbi.nlm.nih.gov/ pubmed/22563003 (Accessed: 16, July, 2012).

Ward, B. D. (2000). Simultaneous Inference for fMRI Data. Biophysics Research Institute,Medical College of Wisconsin. Available online at: http://afni.nimh.nih.gov/pub/ dist/doc/manual/AlphaSim.pdf.

Wechsler, D. (1999). Manual for the Wechsler Abbreviated Intelligence Scale (WASI). San Antonio, TX: The Psychological Corporation.

Weiss, L. G., Saklofske, D. H., Prifitera, A., Chen, H.-Y., and Hildebrand, D. (1999). The calculation of the WISC-Ill general ability index using Canadian norms. Can. J. Sch. Psychol. 14, 1-10. doi: 10.1177/082957359901400202

Weng, S.-J., Wiggins, J. L., Peltier, S. J., Carrasco, M., Risi, S., Lord, C., et al. (2010). Alterations of resting state functional connectivity in the default network in adolescents with autism spectrum disorders. Brain Res. 1313, 202-214. doi: 10.1016/j. brainres.2009.11.057

Wiggins, J. L., Peltier, S. J., Ashinoff, S., Weng, S.-J., Carrasco, M., Welsh, R. C., et al. (2011). Using a selforganizing map algorithm to detect age-related changes in functional connectivity during rest in autism spectrum disorders. Brain Res. 1380,
187-197. doi: 10.1016/j.brainres. 2010.10.102

Wise, R. G., Ide, K., Poulin, M. J., and Tracey, I. (2004). Resting fluctuations in arterial carbon dioxide induce significant low frequency variations in BOLD signal. Neuroimage 21, 1652-1664. doi 10.1016/j.neuroimage.2003.11.025

Yerys, B. E., Wallace, G. L., Harrison, B., Celano, M. J., Giedd, J. N., and Kenworthy, L. E. (2009a). Set-shifting in children with autism spectrum disorders: reversal shifting deficits on the Intradimensional/Extradimensional Shift Test correlate with repetitive behaviors. Autism 13, 523-538.

Yerys, B. E., Wallace, G. L., Sokoloff, J. L., Shook, D. A., James, J. D., and Kenworthy, L. (2009b). Attention deficit/hyperactivity disorder symptoms moderate cognition and behavior in children with autism spectrum disorders. Autism Res. 2, 322-333.

Zink, C. F., Pagnoni, G., Martin, M. E., Dhamala, M., and Berns, G. S. (2003). Human striatal response to salient nonrewarding stimuli. J. Neurosci. 23, 8092-8097.

Conflict of Interest Statement: The authors declare that the research was conducted in the absence of any commercial or financial relationships that could be construed as a potential conflict of interest.

Received: 03 June 2013; accepted: 30 July 2013; published online: 27 August 2013. Citation: You X, Norr M, Murphy E, Kuschner ES, Bal E, Gaillard WD, Kenworthy L and Vaidya CJ (2013) Atypical modulation of distant functional connectivity by cognitive state in children with Autism Spectrum Disorders. Front. Hum. Neurosci. 7:482. doi: 10.3389/fnhum.2013.00482

This article was submitted to the journal Frontiers in Human Neuroscience.

Copyright (c) 2013 You, Norr, Murphy, Kuschner, Bal, Gaillard, Kenworthy and Vaidya. This is an open-access article distributed under the terms of the Creative Commons Attribution License (CC BY). The use, distribution or reproduction in other forums is permitted, provided the original author(s) or licensor are credited and that the original publication in this journal is cited, in accordance with accepted academic practice. No use, distribution or reproduction is permitted which does not comply with these terms. 